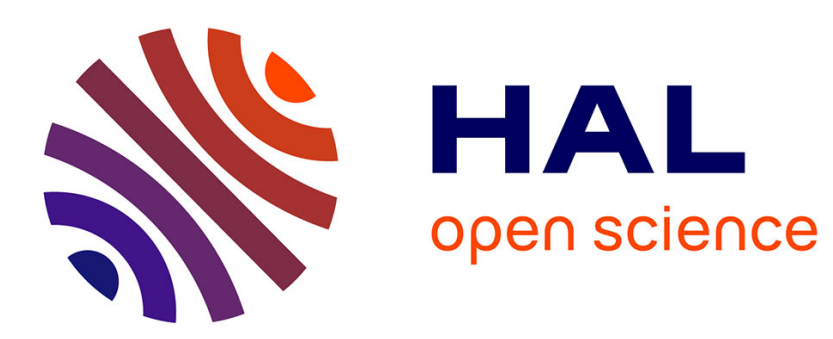

\title{
Quantum cloning and teleportation criteria for continuous quantum variables
}

\author{
Frédéric Grosshans, Philippe Grangier
}

\section{To cite this version:}

Frédéric Grosshans, Philippe Grangier. Quantum cloning and teleportation criteria for continuous quantum variables. Physical Review A : Atomic, molecular, and optical physics [1990-2015], 2001, 64, pp.010301(R). 10.1103/PhysRevA.64.010301 . hal-00509127

\section{HAL Id: hal-00509127 \\ https://hal.science/hal-00509127}

Submitted on 14 Apr 2016

HAL is a multi-disciplinary open access archive for the deposit and dissemination of scientific research documents, whether they are published or not. The documents may come from teaching and research institutions in France or abroad, or from public or private research centers.
L'archive ouverte pluridisciplinaire HAL, est destinée au dépôt et à la diffusion de documents scientifiques de niveau recherche, publiés ou non, émanant des établissements d'enseignement et de recherche français ou étrangers, des laboratoires publics ou privés. 


\title{
Quantum cloning and teleportation criteria for continuous quantum variables
}

\author{
Frédéric Grosshans and Philippe Grangier \\ Laboratoire Charles Fabry, Institut d'Optique Théorique et Appliquée, F-91403 Orsay, France
}

(Received 21 December 2000; published 12 June 2001)

\begin{abstract}
We discuss the criteria presently used for evaluating the efficiency of quantum teleportation schemes for continuous variables. Using an argument based upon the difference between 1-to-2 quantum cloning (quantum duplication) and 1-to-infinity cloning (classical measurement), we show that a fidelity value larger than $2 / 3$ warrants that the teleported state is the best possible remaining copy of the input state. This value has not been reached experimentally so far.
\end{abstract}

DOI: 10.1103/PhysRevA.64.010301

PACS number(s): 03.67.- a, 42.50.Dv, 03.65.Ta, 89.70.+c

\section{INTRODUCTION}

Quantum teleportation has emerged in recent years as a major paradigm of theoretical [1] and experimental [2,3] quantum information. The initial approaches using discrete variables $[1,2]$ have been extended to continuous quantum variables [3-7]. However, various discussions have appeared recently about the significance and the evaluation criteria of real, and thus imperfect, teleportation experiments [5-11]. In this article, we will reconsider the teleportation criteria for continuous quantum variables, with emphasis on the teleportation of coherent states [3]. Using an argument based upon the no-cloning theorem $[12,13]$, we will show that a teleportation fidelity $F_{t e l}>2 / 3$ warrants that the teleported state is the best remaining copy of the input state.

In order to set the scene, it may be useful to come back to [1], where Bennett et al. introduce and define the concept of quantum teleportation. This quotation is taken from their paper: "Below, we show how Alice can divide the full information encoded in [the unknown quantum state] $|\phi\rangle$ into two parts, one purely classical and the other purely non-classical, and send them to Bob through two different channels. Having received these two transmissions, Bob can construct an accurate replica of $|\phi\rangle$. Of course Alice's original $|\phi\rangle$ is destroyed in the process, as it must be to obey the no-cloning theorem. We call the process we are about to describe teleportation, a term of science-fiction meaning to make a person or object disappear while an exact replica appears somewhere else.'

From this definition it should be clear that teleportation must not only beat the classical limits on measurement and transmission, but must also reach the limit where the nocloning theorem is enforced, otherwise Bob may receive a state that is better than any classical copy, but nevertheless it will not be the teleported $|\phi\rangle$. A crucial point is then that there is a distinction between nonclonable quantum information and classical information. This is best illustrated by considering the fidelity for cloning one copy of a coherent state into $M$ identical copies, which is $F_{1 \rightarrow M}=2 /\left(2+N^{a d}\right)$, where $N^{a d}=2(M-1) / M$, as shown by Cerf and Iblisdir [13] $\left(N^{a d}\right.$ is an equivalent noise in the cloning process [9], that will be discussed in more detail below). It is then clear that $F_{1 \rightarrow \infty}$ $=1 / 2$, while $F_{1 \rightarrow 2}=2 / 3$. The usual criterion about teleportation assumes correctly that a classical measurement is involved in teleportation. However, it should not be concluded that the relevant limit is the one associated with a classical measurement, $F_{1 \rightarrow \infty}=1 / 2$. This conclusion is incorrect because the good question to ask is: what is the measured fidelity of Bob's copy, as measured by the verifier Victor, which warrants that no better copy of the input state can exist elsewhere? (i.e., kept by a cheating Alice, or eavesdropped by a malicious Eve). We will show below in detail, but it should already be clear from the above cloning limit, that the correct answer is $F_{1 \rightarrow 2}=2 / 3$.

\section{THE $1 \rightarrow 2$ AND $1 \rightarrow M$ CLONING LIMITS}

\section{A. Quantum duplication}

We first give a simple demonstration that the fidelity limit for making two copies of an input state is $F_{1 \rightarrow 2}=2 / 3$, as it was previously shown by Cerf et al. in [12]. Here we recover the same conclusion by using simple techniques similar to the ones used for evaluating quantum nondemolition (QND) measurements, introduced in [14-16] and used in $[6,9,10]$.

A $1 \rightarrow 2$ cloner or "duplicator" has one input mode and two output modes $a$ and $b$. Denoting by $g$ and $B$ the (linearized) gains and noises for each channel, the quadratures of the two output modes are related to the two input quadratures $X_{\text {in }}$ and $Y_{\text {in }}$ by

$$
\begin{array}{ll}
X_{a}=g_{X_{a}} X_{i n}+B_{X_{a}}, & Y_{a}=g_{Y_{a}} Y_{i n}+B_{Y_{a}} \\
X_{b}=g_{X_{b}} X_{i n}+B_{X_{b}}, & Y_{b}=g_{Y_{b}} Y_{i n}+B_{Y_{b}} .
\end{array}
$$

Since $a$ and $b$ are two different field modes, any observable of $a$ commutes with any observable of $b$, and in particu$\operatorname{lar}\left[X_{a}, Y_{b}\right]=0$. Using Eqs. (1), and assuming that the added noises are not correlated to the input signals, we obtain

$$
\left[B_{X_{a}}, B_{Y_{b}}\right]=-g_{X_{a}} g_{Y_{b}}\left[X_{i n}, Y_{i n}\right]
$$

The noises added by the duplicator verify therefore

$$
\Delta B_{X_{a}} \Delta B_{Y_{b}} \geqslant\left|g_{X_{a}} g_{Y_{b}}\right| N_{0},
$$

where $N_{0}$ is the vacuum's noise variance and $\Delta$ denotes the usual rms dispersion.

It is convenient to define the variances of the equivalent input noises [15] associated with the measurements

$$
N_{X_{i}}=\left(\Delta X_{i} /\left|g_{X_{i}}\right|\right)^{2}-\left(\Delta X_{i n}\right)^{2}=\left(\Delta B_{X_{i}} /\left|g_{X_{i}}\right|\right)^{2},
$$




$$
N_{Y_{i}}=\left(\Delta Y_{i} /\left|g_{Y_{i}}\right|\right)^{2}-\left(\Delta Y_{i n}\right)^{2}=\left(\Delta B_{Y_{i}} /\left|g_{Y_{i}}\right|\right)^{2},
$$

where $i$ is either $a$ or $b$. One thus obtains the symmetrical inequalities

$$
N_{X_{a}} N_{Y_{b}} \geqslant N_{0}^{2}, \quad N_{X_{b}} N_{Y_{a}} \geqslant N_{0}^{2} .
$$

These inequalities are very similar to the ones that appear in QND measurements [15], and they ensure that building two copies of the input state will not allow one to work around the Heisenberg inequality. Actually, the added noise is just the one required to forbid to infer the values of $X_{i n}$ and $Y_{\text {in }}$ with a precision better than Heisenberg limit, by measuring $X_{a}$ and $Y_{b}$.

The equivalent noises can be easily related to the cloning fidelity. It can be shown straightforwardly $[9,10]$ that the fidelity obtained when copying coherent states with unity gain $\left(g_{X_{i}}=g_{Y_{i}}=1\right)$ is given by

$$
F_{g_{T}=1}=\frac{2}{\sqrt{\left(2+N_{X} / N_{0}\right)\left(2+N_{Y} / N_{0}\right)}} .
$$

Assuming that the two copies are identical and have phaseindependent noise, the limit of Eq. (5) is reached for $N_{X_{a}}$ $=N_{Y_{b}}=N_{X_{b}}=N_{Y_{a}}=N_{0}$ and corresponds thus to $F_{g_{T}=1}$ $=2 / 3$. This is identical to the result obtained by Cerf et al. in [12]. A "duplicator' reaching the limit of Eq. (5), can be easily implemented using a linear amplifier and a 50/50 beam splitter. Such a duplicator is a Gaussian cloning machine as defined by Cerf et al. [12]. Various implementations of "cloners"' have been proposed recently [17], and may allow, in particular, to arbitrarily share the noise between one copy that is kept, and another one that is sent out.

\section{B. The $1 \rightarrow M$ cloning limit}

We generalize here the above demonstration to copying one input to $M$ identical outputs. In order to directly recover the result of Cerf and Iblisdir [13], we will assume that each output channel has unity gain, and that all copies are identical in the sense that the variances are the same for all output, and that the pairwise correlation does not depend on the pair of outputs that is considered. More precisely, the quadratures of the $M$ outputs of a $1 \rightarrow M$ cloner $(M>2)$ obey

$$
\begin{aligned}
& X_{i}=X_{i n}+B_{X_{i}} \\
& Y_{i}=Y_{i n}+B_{Y_{i}},
\end{aligned}
$$

for every $1 \leqslant i \leqslant M$. We define $C_{X}, N_{X}$, and $N_{Y}$ as

$$
\begin{gathered}
C_{X}=\left\langle B_{X_{i}} B_{X_{j}}\right\rangle \text { for every } i \neq j, \\
N_{X}=\Delta B_{X_{i}}^{2} \text { for every } i, \\
N_{Y}=\Delta B_{Y_{i}}^{2} \text { for every } i .
\end{gathered}
$$

Like in Sec. II A, we have

$$
\begin{gathered}
{\left[B_{X_{i}}, B_{Y_{j}}\right]=-\left[X_{i n}, Y_{i n}\right] \text { for every } i \neq j,} \\
{\left[B_{X_{i}}, B_{Y_{i}}\right]=0 \text { for every } i .}
\end{gathered}
$$

We can define $\Lambda$ for any real number $\lambda$ by

$$
\Lambda=B_{X_{1}}+\lambda \sum_{i=2}^{M} B_{X_{i}}
$$

It follows straightforwardly from Eqs. (9) and (10), that

$$
\left[\Lambda, B_{Y_{1}}\right]=-\lambda(M-1)\left[X_{i n}, Y_{i n}\right]
$$

For the variances, it implies

$$
\Delta \Lambda \Delta B_{Y_{1}} \geqslant\left|\lambda(M-1) N_{0}\right|
$$

Computing $\Delta \Lambda^{2}$ directly from Eq. (11), we have

$$
\begin{aligned}
\Delta \Lambda^{2}= & \Delta B_{X_{1}}^{2}+\lambda^{2} \sum_{i=2}^{M} \Delta B_{X_{i}}^{2}+2 \lambda \sum_{i=2}^{M}\left\langle B_{X_{1}} B_{X_{i}}\right\rangle \\
& +\lambda^{2} \sum_{i, j>1}^{i \neq j}\left\langle B_{X_{i}} B_{X_{j}}\right\rangle .
\end{aligned}
$$

Using the definitions (8) in Eq. (14), we obtain

$$
\begin{aligned}
\Delta \Lambda^{2}= & {\left[1+\lambda^{2}(M-1)\right] N_{X} } \\
& +\left[2 \lambda(M-1)+\lambda^{2}(M-1)(M-2)\right] C_{X} .
\end{aligned}
$$

If $\lambda=-2 /(M-2)$, this expression is simpler and becomes

$$
\Delta \Lambda^{2}=\frac{M^{2}}{(M-2)^{2}} N_{X},
$$

which can be injected in Eq. (13) to obtain the $1 \rightarrow M$ cloning limit

$$
N_{X} N_{Y} \geqslant\left(\frac{2(M-1)}{M}\right)^{2} N_{0}^{2} .
$$

This limit is also valid for $M=2$ as written in Eq. (5) and for the trivial case $M=1$.

Assuming that the $M$ copies have phase-independent noise, i.e., $N_{X} / N_{0}=N_{Y} / N_{0}=N^{a d}=2(M-1) / M$, it is simple to show from Eq. (6) that the corresponding fidelity limit for coherent state cloning is

$$
F_{1 \rightarrow M}=\frac{2}{2+N^{a d}} \leqslant \frac{M}{2 M-1} .
$$

As previously stated, a perfect $1 \rightarrow M$ symmetrical cloner can be implemented using a linear amplifier and $M-1$ beam splitters [17].

\section{The $1 \rightarrow \infty$ cloning and classical measurements}

When a classical measurement is performed, the measurement result can be copied an arbitrary number of times. It 
should thus be clear that the limit corresponding to a classical measurement is $F_{1 \rightarrow \infty}=1 / 2$, or $N^{a d}=2$. On the other hand, making only two copies comes at a smaller price, and corresponds to $F_{1 \rightarrow 2}=2 / 3$, or $N^{a d}=1$. We will show below that this distinction is crucial as far as quantum teleportation is concerned.

\section{TELEPORTATION AND NO-CLONING}

\section{A. Quantum teleportation criteria}

Suppose Alice (a) sends a quantum state to Bob (b), who wants to be certain that Alice cannot have kept a better copy of the input state than the one she has given to him. This requirement means to be sure that Alice's copy is destroyed, i.e., that quantum teleportation according to [1] has occurred. Alice will be able to cheat if her equivalent noise is smaller than Bob's, that is,

$$
N_{X_{b}} \geqslant N_{X_{a}}^{o p t} \text { and } N_{Y_{b}} \geqslant N_{Y_{a}}^{o p t}
$$

where opt denotes the optimum result for Alice. Since the best Alice can do is limited by the Heisenberg-like inequalities (5), one has

$$
N_{X_{b}} \geqslant N_{0}^{2} / N_{Y_{b}} \quad \text { and } N_{Y_{b}} \geqslant N_{0}^{2} / N_{X_{b}}
$$

and thus

$$
N_{X_{b}} N_{Y_{b}} \geqslant N_{0}^{2}
$$

If Bob's noise variances are symmetrical, i.e., $N_{X_{b}}=N_{Y_{b}}$, one recovers the limit $F \leqslant 2 / 3$ for teleporting coherent states. Thus the only way for Victor to warrant that Alice is not cheating is to obtain a measured teleportation fidelity larger that $2 / 3$. It is worth noticing that when the associated condition $N_{X_{b}} N_{Y_{b}}<N_{0}^{2}$ is fulfilled, then Eq. (5) imposes that $N_{X_{b}}$ $<N_{X_{a}}$ and $N_{Y_{b}}<N_{Y_{a}}$, and thus Alice will have both quadratures worse than Bob.

\section{B. Security in quantum teleportation}

It should be clear now that as long as $F \leqslant 2 / 3$, Alice can cheat teleportation by keeping a better copy than the one Bob has received. The simplest way to do that is first to duplicate the input state, then to keep one copy, and to teleport the other one to Bob. As an example, if Bob's teleported output has a fidelity $F_{b}=0.58$, or $N_{b}=1.45$, and if Alice has a perfect teleporter than she claims to be imperfect, she can keep a copy with a fidelity $F_{a}=0.74$, or $N_{a}=0.7$. This is clearly not acceptable according to the definition of [1].

We point out that the same condition applies when Alice is honest, but when quantum teleportation is used to send a quantum state from Alice to Bob for quantum cryptography purposes. In that case, one must worry about the amount of information that can be eavesdropped during the teleportation process. For simplicity, let us consider a teleportation scheme using light beams exhibiting Einstein-PodolskyRosen (EPR) correlations, with a finite degree of squeezing, and transmission losses. It is assumed that Eve is able to

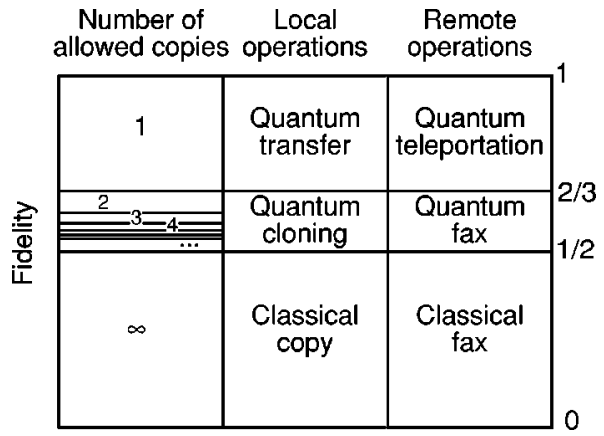

FIG. 1. Table illustrating the fidelity values associated to the number of allowed copies of the input state. The value $F=1 / 2$ corresponds to the threshold for the use of quantum entanglement, while the value $F=2 / 3$ corresponds to the enforcement of nocloning. For distant operations, the region between $F=1 / 2$ and $F$ $=2 / 3$ is called "quantum fax," because the original kept by Alice may have a higher fidelity than the teleported copy. The no-cloning region $F>2 / 3$ corresponds to tranferring quantum states between different systems for local operations, and to quantum teleportation (as defined in [1]) for remote operations.

perfectly eavesdrop the classical channel, and that she has full access to the losses along at least one "transmission arm" of the EPR beam (this is a strong hypothesis, but it is usually done for evaluating the security of standard quantum cryptography). The simplest solution for Eve is to build her own teleported state, and she will be successful if this state has an equivalent noise smaller than the one achieved by Bob. It can be shown simply, and it is physically obvious, that as long as the EPR channel efficiency $\eta$ is smaller than $1 / 2$, Eve can obtain a teleported copy of the input state that is better than the one obtained by Bob. More generally, this can be also seen as a consequence of the $1 \rightarrow 2$ cloning limit: if $F$ is larger than 2/3, Bob can be sure that a malicious Eve will not be able to eavesdrop the teleported state [10]. Thus the $F>2 / 3$ limit appears also as a crucial security condition if teleportation is used as a quantum communication tool.

\section{Discussion}

In order to clarify the issues involved, it may be worth summarizing the physics involved in the respective criteria $F>1 / 2$ and $F>2 / 3$.

As said above, $F=1 / 2$ is actually a classical measurement limit, associated with the $1 \rightarrow \infty$ cloning limit. It has been shown in [18] that purifications procedure can be initiated as soon as $F>1 / 2$, and may lead to high fidelity values. However, the purpose of teleportation criteria is to characterize a given experiment, and not what it might be by adding purification procedures. We note also that recently demonstrated entanglement criteria [19] are fully compatible with the $F$ $=1 / 2$ limit. It is thus clear that the $F>1 / 2$ criterion characterizes a threshold for the appearance of quantum effects, related to entanglement in the teleportation process $[5,7]$.

On the other hand, the main virtue of the $F>2 / 3$ criterion is that it warrants that no other copy of the input state can remain, that would have a better fidelity than the one Bob has received. This has obvious advantages if quantum tele- 
portation is used as a secure way to transmit quantum information. It is worth pointing out that the $F>2 / 3$ criterion is more demanding technically, and can be fulfilled only when the transmission of each beam distributing the entanglement resource is higher than $50 \%$, and enough squeezing is available $[9,10,20]$.

In $[9,10]$ it was argued that the $F>2 / 3$ criterion is also related to the so-called EPR nonseparability argument, which was introduced in [21] and implemented experimentally in [22]. This argument requires that "conditional squeezing"' is obtained on one EPR beam, given a measurement that is done on its entangled partner. By changing the measurement, one can get conditional squeezing in both quadratures, creating an apparent violation of Heisenberg's relations [21,22]. It was shown in [9] that $F>2 / 3$ is a sufficient condition to warrant that such conditional squeezing can be obtained on the EPR beams. Though the status of this EPR-Heisenberg argument is a subject of debates [9-11], these various remarks strongly suggest that the $F=2 / 3$ limit is related to inferences made using conditional measurements, which play an essential role both for QND measurements [14-16] and for the noncloning theorem $[12,13]$.

Finally, the status of the local or remote operations that can be performed on the input state is summarized in Fig. 1. The wording "quantum fax" for remote operations with a fidelity between $1 / 2$ and $2 / 3$ means that a quantum entangle- ment resource must be used to reach that region, but nevertheless that the no-cloning theorem is not yet enforced. Therefore, as in a fax machine, Bob has received something that is not so bad, but a better copy may still exist somewhere. Obviously, this does not happen any more in the region above $F=2 / 3$.

\section{CONCLUSION}

As a conclusion, it should be clear that the criteria $F$ $>1 / 2$ and $F>2 / 3$ have different physical contents, and are both legitimate. Based upon the definition given in [1], and on the no-cloning theorem, we showed that in order to warrant the destruction of the initial state one should require $F$ $>2 / 3$. However, it should be clear that though the result $F_{\text {exp }}=0.58$ reported in Ref. [3] falls below that value, this experiment is nevertheless a very significant achievement in defining and using the concept of continuous variables quantum teleportation.

\section{ACKNOWLEDGMENTS}

This work was carried out in the framework of the European IST/FET/QIPC project "QUICOV.', Laboratoire Charles Fabry is CNRS UMR 8501.
[1] C. H. Bennett et al., Phys. Rev. Lett. 70, 1895 (1993).

[2] D. Bouwmeester et al., Nature (London) 390, 575 (1997); D. Boschi et al., Phys. Rev. Lett. 80, 1121 (1998).

[3] A. Furusawa et al., Science 282, 706 (1998).

[4] L. Vaidman, Phys. Rev. A 49, 1473 (1994).

[5] S. L. Braunstein and H. J. Kimble, Phys. Rev. Lett. 80, 869 (1998); S. L. Braunstein, C. A. Fuchs, and H. J. Kimble, J. Mod. Opt. 47, 267 (2000).

[6] T. C. Ralph and P. K. Lam, Phys. Rev. Lett. 81, 5668 (1998); T. C. Ralph, Opt. Lett. 24, 348 (1999).

[7] L. Vaidman and N. Yoran, Phys. Rev. A 59, 116 (1999); S. M. Tan, ibid. 60, 2752 (1999); S. J. van Enk, ibid. 60, 5095 (1999); P. van Loock and S. L. Braunstein, ibid. 61, 010302 (2000).

[8] S. L. Braunstein and H. J. Kimble, Nature (London) 394, 840 (1998).

[9] P. Grangier and F. Grosshans, e-print quant-ph/0009079.

[10] P. Grangier and F. Grosshans, e-print quant-ph/0010107.

[11] S. L. Braunstein, C. A. Fuchs, H. J. Kimble, and P. van Loock, e-print quant-ph/0012001.
[12] N. J. Cerf, A. Ipe, and X. Rottenberg, Phys. Rev. Lett. 85, 1754 (2000).

[13] N. J. Cerf and S. Iblisdir, Phys. Rev. A 62, 040301 (2000).

[14] M. J. Holland, M. J. Collett, D. F. Walls, and M. D. Levenson, Phys. Rev. A 42, 2995 (1990).

[15] J.-Ph. Poizat, J.-F. Roch, and P. Grangier, Ann. Phys. (Paris) 19, 265 (1994).

[16] Ph. Grangier, J.-A. Levenson, and J.-Ph. Poizat, Nature (London) 396, 537 (1998).

[17] For several recent proposals of cloning devices see e.g., Phys. Rev. Lett. 86, 914 (2001); e-print quant-ph/0012025; e-print quant-ph/0012046; e-print quant-ph/0012048.

[18] L.-M. Duan et al., Phys. Rev. Lett. 84, 4002 (2000).

[19] L.-M. Duan et al., Phys. Rev. Lett. 84, 2722 (2000); R. Simon, ibid. 84, 2726 (2000).

[20] J. Mertz et al., Phys. Rev. A 44, 3229 (1991).

[21] M. D. Reid and P. D. Drummond, Phys. Rev. Lett. 60, 2731 (1988); M. D. Reid, Phys. Rev. A 40, 913 (1989).

[22] Z. Y. Ou et al., Phys. Rev. Lett. 68, 3663 (1992); Z. Y. Ou et al., Appl. Phys. B: Photophys. Laser Chem. 55, 265 (1992). 